\title{
条的 J.B.METZLER
}

Ergänzende Unterlagen zum Buch bieten wir Ihnen unter www.metzlerverlag.de/webcode zum Download an.

Für den Zugriff auf die Daten verwenden Sie bitte Ihre E-Mail-Adresse und Ihren persönlichen Webcode. Bitte achten Sie bei der Eingabe des Webcodes auf eine korrekte Groß- und Kleinschreibung.

Ihr persönlicher Webcode: 
Hans-Jörg Neuschäfer

\section{Klassische Texte der spanischen Literatur}

25 Einführungen vom Cid bis Corazón tan blanco

Verlag J. B. Metzler

Stuttgart - Weimar 
Bibliografische Information der Deutschen Nationalbibliothek

Die Deutsche Nationalbibliothek verzeichnet diese Publikation in der Deutschen Nationalbibliografie; detaillierte bibliografische Daten sind im Internet über http://dnb.d-nb.de abrufbar.

ISBN 978-3-476-02397-1

ISBN 978-3-476-05277-3 (eBook)

DOI 10.1007/978-3-476-05277-3

Dieses Werk einschließlich aller seiner Teile ist urheberrechtlich geschützt. Jede Verwertung außerhalb der engen Grenzen des Urheberrechtsgesetzes ist ohne Zustimmung des Verlages unzulässig und strafbar. Das gilt insbesondere für Vervielfältigungen, Übersetzungen, Mikroverfilmungen und die Einspeicherung und Verarbeitung in elektronischen Systemen.

(C) 2011 Springer-Verlag GmbH Deutschland

U rsprünglich erschienen bei J. B. Metzler'sche Verlagsbuchhandlung und Carl Ernst Poeschel Verlag GmbH in Stuttgart 2011

www.metzlerverlag.de info@metzlerverlag.de 


\section{Inhalt}

Verzeichnis der ergänzenden Unterlagen zum Download ............. VIII

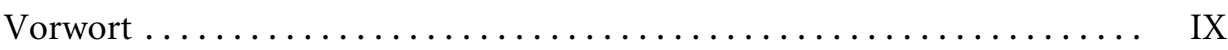

\section{Kapitel I}

El Cantar de Mio Cid (um 1200)

Reconquista und Castellanocentrismo ....................... 1

\section{Kapitel II}

El libro de buen amor (1330/1343)

Der Erzpriester von Hita und die `menschliche Natur $\ldots . . \ldots \ldots \ldots \ldots . \quad 10$

\section{Kapitel III}

Der Romancero als Unterhaltungsliteratur (ab 15. Jh.) ............ 20

\section{Kapitel IV}

Fernando de Rojas: La Celestina (1499)

Die Entdeckung der Sexualität

\section{Kapitel V}

Lazarillo de Tormes (1554)

Die Geburt des Schelmenromans ...

\section{Kapitel VI}

San Juan de la Cruz, Fray Luis de León (16. Jh.)

Das Erlebnis der unio mystica.

\section{Kapitel VII}

Cervantes: Don Quijote (1605/15)

Der Ursprung des modernen Romans .

\section{Kapitel VIII}

Lope de Vega: Fuenteovejuna (1610)

Die Kunst des engineering of consent ....................... 65

\section{Kapitel IX}

Calderón: La dama duende (1629)

Frauenwitz und Ehrenmänner 
VI Inhalt

\section{Kapitel X}

Calderón: La vida es sueño (1635)

Lehrstück und barockes Wortkunstwerk

\section{Kapitel XI}

Moratín: El sí de las niñas (1806)

Von der Schwierigkeit, eine aufklärerische Komödie zu schreiben....... 90

\section{Kapitel XII}

Mariano José de Larra: Artículos de costumbres (1828ff.)

Die Verzweiflung an der Rückständigkeit Spaniens

\section{Kapitel XIII}

José de Espronceda: Canciones (1835/40)

Politische Lyrik in der spanischen Romantik

\section{Kapitel XIV}

José Zorrilla: Don Juan Tenorio (1844)

Der gezähmte Burlador

\section{Kapitel XV}

Benito Pérez Galdós: Tristana (1892)

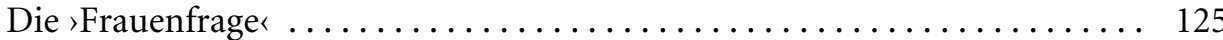

\section{Kapitel XVI}

Clarín: La Regenta (1885)

Der Abscheu vor Vetusta.

\section{Kapitel XVII}

Antonio Machado: Campos de Castilla (1912/1917)

Die Poesie der Alltäglichkeit

\section{Kapitel XVIII}

Unamuno: Niebla (1914)

Auf der Suche nach dem verantwortlichen Autor.

\section{Kapitel XIX}

Valle-Incláns Luces de Bohemia (1921/24)

Spanien als Esperpento

\section{Kapitel XX}

Rafael Alberti: Cal y Canto (1926/27)

Modernisierter Gongorismus 


\section{Kapitel XXI}

Miguel Mihura: Tres sombreros de copa (1932/52)

Die Erfindung des absurden Theaters

\section{Kapitel XXII}

Federico García Lorca: La casa de Bernarda Alba (1936)

Die Enttabuisierung der weiblichen Sexualität

\section{Kapitel XXIII}

Camilo José Cela: La familia de Pascual Duarte (1942)

Provokation und Besänftigung der Zensur

\section{Kapitel XXIV}

Javier Marías: Corazón tan blanco (1992)

Die Kunst der Fälschung . . .

\section{Kapitel XXV}

Miguel Delibes: El hereje (1998)

Die spanische Vergangenheitsbewältigung 


\section{Verzeichnis der ergänzenden Unterlagen zum Download}

Dieses Buch bietet die Möglichkeit, zusätzliche Materialien auf dem Weg eines Downloads von der Website des Verlages zu erhalten. Es sind dies Texte, die alle dem folgenden Werk entnommen sind:

Hans-Jörg Neuschäfer (Hrsg.), Spanische Literaturgeschichte.

Unter Mitarbeit von Sebastian Neumeister, Gerhard Poppenberg, Jutta Schütz und Manfred Tietz.

4., aktualisierte und erweiterte Auflage. Stuttgart/Weimar 2011.

Folgende Texte sind verfügbar:

1. Bibliographie zur spanischen Literaturgeschichte

2. Personen- und Werkregister (mit Lebensdaten)

3. Zu den Anfängen der spanischen Literatur (Manfred Tietz)

4. Tendenzen der spanischen Gegenwartsliteratur (Hans-Jörg Neuschäfer) 


\section{Vorwort}

Dieses Buch ist eine Hommage an die spanische Literatur und zugleich eine Handreichung für Studierende, für Lehrerinnen und Lehrer an den Schulen und Hochschulen und für Spanienliebhaber, kurz für alle, die sich für die Literatur des Landes interessieren und gern etwas über deren herausragende Texte erfahren möchten, ohne sie in toto lesen zu können, sei es, weil sie sich in ihrer Ausbildung einschlägige Kenntnisse überhaupt erst aneignen müssen; sei es, weil sie früher Gelesenes inzwischen vergessen oder nicht mehr wirklich präsent haben; sei es auch, weil sie ganz einfach neugierig sind auf das, was sie schon immer gern wissen wollten, aber nicht in Erfahrung bringen konnten, weil Lexika (gedruckte wie elektronische) zu knappe und wissenschaftliche Abhandlungen zu spezielle Auskunft geben. Diesen Interessierten werden hier, aus einer Hand, 25 Einführungen in ebenso viele Haupttexte der spanischen Literatur geboten; Einführungen, denen sie nicht nur entnehmen können, um was es in diesen Texten inhaltlich geht und wie sie formal gestaltet sind. Sie werden auch darüber informiert, aus welchem Anlass und in welchem Kontext sie entstanden sind; auf welche Zeitumstände sie Rücksicht nahmen und was uns heute gleichwohl noch mit ihnen verbindet. Es wäre schön, wenn sich die Benutzer selbst zum Lesen verführen ließen, am besten im Original. Zur Beförderung dieses Zwecks gibt es in jedem Kapitel eine oder mehrere Leseproben, dazu eine deutsche Übersetzung. Wenn man will, kann man das vorliegende Buch also selbst schon als Lesebuch betrachten, auch wenn die meisten es wohl eher als ein hoffentlich angenehm zu benutzendes Nachschlagewerk gebrauchen werden. Der Charakter des Lesebuchs wird übrigens auch dadurch unterstrichen, dass der laufende Text keine Fußnoten enthält.

Ich kenne den Einwand, man solle es vor allem den Studierenden nicht so leicht machen. Aber Hand aufs Herz: Wer kennt schon alles, was er kennen sollte? Das müssen sich gelegentlich selbst Spezialisten auf ihrem engeren Fachgebiet fragen. Dass wir manchmal über Bücher reden, die wir gar nicht gelesen haben, hat unlängst Pierre Bayard - selbst ein Leseprofi - in seinem ebenso vergnüglichen wie bedenkenswerten Essay Comment parler des livres que l'on a pas lus? (2007, im selben Jahr auch auf Deutsch erschienen) nicht nur eingestanden, sondern mit guten Gründen auch noch verteidigt. Wir müssen uns halt dort, wo wir (noch) nicht selbst Bescheid wissen, auf andere verlassen, die in diesem Fall Auskunft erteilen können.

Die Auswahl der 25 Texte, die ich dem Leser vorstelle, war insofern weitgehend vorgegeben, als es sich in ihrer überwiegenden Mehrzahl um kanonisierte, durch eine lange Tradition als unverzichtbar gekennzeichnete Texte handelt, über deren exemplarische Bedeutung Konsens herrscht. Insoweit hat das Buch bewahrenden Charakter. Es spiegelt auch mit voller Absicht die Lektürelisten für Studierende der 
Hispanistik wider; schließlich soll es ja nützlich sein. Die Texte selbst aber, die das kollektive Gedächtnis aufbewahrt und weiter tradiert hat, stehen unter einer ganz besonderen Spannung: Sie tragen einerseits den Stempel ihrer Entstehungszeit und sprengen andererseits doch auch den Rahmen dessen, was in ihr üblich war oder als Norm galt. Und eben diese Dialektik von Zeitbedingtheit und Zeitüberwindung macht ihre Faszination aus.

Je näher man der Gegenwart kommt, desto unbestimmter und subjektiver wird allerdings die Vermutung, ein Text könne die Zeiten überdauern. Da es aber in der vorliegenden Publikation nur um längerfristig wirksame Texte geht, habe ich keinen Titel aus dem neuen Jahrhundert aufgenommen: Sie sind noch zu >frisch und müssen erst noch zeigen, ob sie über den Tag hinaus gültig bleiben, an dem sie von immer aufdringlicher werdenden Marketingstrategen als >unübertrefflich lanciert wurden. Nur in zwei Fällen - Miguel Delibes und Javier Marías - habe ich neuere Texte (aus den 1990er Jahren) ausgewählt, von denen ich meine, sie hätten die Nachhaltigkeitsprobe bereits bestanden. Wer Näheres über die spanische Literatur der Gegenwart erfahren möchte, sei auf deren umfangreiche Darstellung in der von mir herausgegebenen Spanischen Literaturgeschichte verwiesen, die gleichzeitig mit dem vorliegenden Band in der erweiterten vierten Auflage bei Metzler erscheint.

Der Begriff des kanonischen Textes ist nicht unproblematisch. Er gehört, insofern er sich eigentlich auf die Texte der Heiligen Schrift bezieht, in den Bereich der Theologie, ja der Glaubenslehre. Literarische Texte sind aber keine heiligen, sondern ganz im Gegenteil profane und infolgedessen oft auch stark umstrittene Texte, an denen man sich erfreuen und von denen man sich belehren lassen kann, an die man aber nicht sglauben < muss. Es gab einmal eine - vom deutschen Idealismus geprägte - Zeit, wo die Texte der `Klassiker` quasi als säkularisierte Heilsbringer betrachtet wurden, aber diese Zeit ist längst vorbei. Deshalb werden die hier behandelten Texte nicht kritiklos gepriesen, sondern in ihrer historischen Bedingtheit betrachtet, auch und gerade in der Spannung zwischen ihrer einstigen und ihrer jetzigen Bedeutung.

Selbstverständlich gibt es in der fast tausendjährigen Geschichte der spanischen Literatur weit mehr als 25 Texte, die als kanonisch gelten dürfen. Die hier zu treffende Auswahl ist deshalb auch durch persönliche Vorlieben mitbestimmt. Und da zudem jedes Kapitel nur einen knapp bemessenen Umfang hat, kann immer nur ein begrenztes Spektrum von Fragestellungen zur Sprache kommen, die nicht immer die gleichen sind: Eine schematische Abarbeitung wäre für Autor und Leser gleichermaßen langweilig. Die Kapitelüberschriften machen jeweils aufmerksam auf das, was neben der in jedem Fall gewährleisteten Textbeschreibung noch besonders berücksichtigt wird.

Zum Schluss noch ein Wort zu den Übersetzungen und zur Bibliographie. Die Übersetzungen beschränken sich auf größere, zusammenhängend dargebotene Passagen. Sie stehen separat, jeweils am Ende des Kapitels. Kleinere, in den laufenden Text eingezogene Originalzitate werden nicht übersetzt. Sie mögen die schon im Spanischen Geübten dazu animieren, das Erlernte auch anzuwenden, und für die 
Ungeübten erklären sie sich aus dem Kontext meines Kommentars. Soweit keine brauchbaren Übertragungen vorhanden waren, habe ich sie selbst gemacht - so nah wie möglich am Wortlaut des Originals.

Am Ende eines jeden Kapitels stehen Hinweise auf die benutzten Ausgaben. Es sind meist solche, die einen Kommentar enthalten. Es schließt sich eine knappe Bibliographie an, die stets mit dem Verweis auf die bereits erwähnte, von mir herausgegebene Spanische Literaturgeschichte, und zwar die 4. Auflage (2011), eröffnet wird. Ergänzendes hispanistisches Basiswissen samt detaillierten bibliographischen Hinweisen wird, außer in der Spanischen Literaturgeschichte, auch in der Einführung in die spanische Literaturwissenschaft von Hartmut Stenzel vermittelt, die im gleichen Verlag erschienen ist. Darüber hinaus sei auf leicht zu erschließende online-Zugänge verwiesen: die von Christoph Strosetzki herausgegebene Bibliographie der deutschen Hispanistik, die Biblioteca virtual Cervantes und den Katalog der Madrider Nationalbibliothek, nicht zu vergessen das speziell die hispanistische Forschung dokumentierende Web-Portal cibera.

Zusätzlich ist noch, per Download auf einen Rechner, ein Blick in die Spanische Literaturgeschichte möglich. Per Mausklick kann man deren fünfzehnseitige, nach Epochen gegliederte Bibliographie, das Personen- und Werkregister (mit Lebensdaten), den Abschnitt »Zu den Anfängen der spanischen Literatur« aus dem Kapitel »Mittelalter und Spätmittelalter « sowie den Abschnitt»1975-2010. Tendenzen der spanischen Gegenwartsliteratur« aus dem Kapitel »Vom 20. Jahrhundert bis zur Gegenwart« anwählen. 